\title{
A Review of Advancements in Robotic and Sensor-based Technologies in Construction Sector
}

\author{
Aditya S. Nath
}

\begin{abstract}
The study explores recent innovations in robotic and sensor-based technologies that are spearheading advancements in the construction sector to achieve improvements in construction quality, efficiency, and safety. Automation in construction, although coined as a concept in 1980s, has witnessed minimal progress in the level of application. The study attempts to identify issues constraining adoption of automation in the sector, the recent developments in technologies and their scope in construction, their applications and impacts, and way forward. The role of robotics in various stages of construction and its impact on a wider scale has been identified and discussed. The evolution of Building Information Modeling (BIM) has transitioned it into being an efficient mediator in the construction process with novel concepts such as 4D and 5D BIM and Building Knowledge Management. Various sensor technologies, functioning at diverse scales, have found wide-ranging applications on construction sites ranging from high-accuracy positioning to slow-tracking of personnel and materials, as well as, in progress monitoring and quality control. Information Technology has a major role in binding the sensor technology with on-site requirements to create positive results. A study was done to identify such technological interventions and various software utilities which integrate BIM and sensor technology with tools such as GIS. The factors which restrained developments in automation in construction sector were identified in the course of study. Various global examples of advanced automated construction technologies with applications in various stages of construction were discussed. The review successfully identifies the nascent technological innovations and their productive usage in relevant areas of construction sector.
\end{abstract}

Keywords - BIM, Construction automation, Construction management, Robotics, Sensors.

\section{INTRODUCTION}

Construction industry has seen minimal change in technology and process over the past century. With technological shifts towards industrial applications in artificial intelligence, machine learning and Internet of Things (IoT) in contemporary fields, various factors have limited evolution in this industry. Its foundation in a craftsmen-based culture, the uniqueness of individual projects and involvement of large execution teams inhibited its evolution. Automation in building industry, in general perception, is connected with robotics. While this holds true, the industry has a potential to be automated well beyond robotics. The industrial age revolutionized construction sector with advent of machinery, which took over bulk physical labour. However, the uniqueness of projects and vast difference in scale prevented standardization, thereby limiting the scope of machinery in the sector. Robotics could, however, effectively substitute human intervention in on-site operations by speeding up construction, reducing errors and working under hazardous conditions. The progress in off-site operations in construction including Building Information Modelling and pre-fabrication technologies could remodel site interventions to minimize labour and save costs. Inefficiency in transfer of knowledge and information across various disciplines of construction is a hurdle that the industry faces which leads to mismanagement issues resulting in wastage of time and resources. Hence efficiency through automation aims at a holistic intervention besides solving day-to-day problems faced in construction sites.

Construction as a collective term involves [1]:

1. Identification of Requirements,

2. Project Planning;

3. Design and Engineering;

4. Construction;

5. Operations and Maintenance;

6. Decommissioning.

The research explores technological interventions in the construction part of the all-inclusive classification.

\section{ThE SCOPE OF AUtOMATION IN CONSTRUCTION}

Standardization due to mass-production is the key factor promoting adoption of automation in major industries. Uniqueness of projects rules out the scope for single algorithm to meet with all production requirements in construction industry. Isolated work packages such as bricklaying and tile fixing are standardized and replicable. The challenge in involving robotic machines to assist on-site labour also lies in the safety of the workers. The major streams in construction where automation through sensorbased technology has an application are:

- Positioning;

- Tracking;

- Progress Monitoring and

- Quality Control.

Automation enhances speed and accuracy. Therefore, automation in positioning, tracking, monitoring and quality control could save time by automated data entry, avoiding mismanagement of data and improving accuracy in data management. 


\section{ROLE OF ROBOTICS}

Robotics is an umbrella term that addresses a variety of devices. An impending change anticipated in building technology is direct involvement of robots in the construction. Robotics will not be used to imitate human methods but to change the construction system to exploit its skills to improve efficiency.

Bock [2] classifies the robotic interventions into five major parts.

\section{A. Robotic-Oriented Design (RoD)}

RoD is a design concept that evolves the conventional building techniques to being more robot-friendly. It involves selection of robots based on their capabilities to achieve a particular function and ensuring favorable conditions, suitable to perform the task.

\section{B. Robotic Industrialization}

Robot industrialization is an initiative to design and perform industrial labor off-site. This includes replicable jobs such as Building Component Manufacturing (BCM) and prefabrication using task-specific robots that can be undertaken off-site.

\section{Construction Robots}

Single Task Construction Robots (STCR) was developed and introduced in construction in 1985. The technology has since remained mostly human-controlled, while developments have occurred in improving speed, accuracy, and strength. The technology foresees a potential to be harnessed with technological developments such as IoT for automated functioning on-site that can contribute further towards reducing human interventions[2].

\section{Site Automation}

The goal of turning construction sites into controlledfactory environments was initiated by STCR. Robotics handle single-objective tasks such as assembly and disassembly of the building components thereby improving material flow and organization in the site. Contour crafting of concrete on site, analogous to 3D printing a model, was performed using a BIM model.

\section{E. Ambient Robotics}

Ambient Robotics deal with predictive maintenance and sustenance of built environment. Being a recent technology, it entered construction industry along with most other fields. This technology could be harnessed to regularize the construction process to improve project monitoring in near future. The technology has potential applications in construction as well as operation and maintenance of buildings [2].

\section{DEVELOPMENTS IN BUILDING INFORMATION MODELING (BIM)}

BIM was developed as a digital representation tool to aid in project management. 4D BIM has an added feature of site scheduling appended to the conventional 3D BIM technology. Embedded with features for material tracking, project monitoring and controlling automated construction labor, 4D BIM can facilitate remote controlling of on-site operations.
According to $\mathrm{Li}$ [3], Internet of Things enabled BIM has been tested successfully by the Hong Kong Housing Authority (HKHA). Ranging from prefabrication to On-site Assembly Services (OAS), The BIM served as a useful platform for the collaborative teamwork. The information regarding materials, additional costs and schedules of prefabrication gets updated on a common platform. Welltimed transfer of materials and equipment within site was ensured using a range of tracking devices including Radiofrequency identification (RFID), Ultra-Wide Band (UWB) and Global Positioning System (GPS.

\section{A. Building Knowledge Management (BKM)}

As discussed, construction scheduling is a novel feature of BIM and is performed by analyzing live-data acquired through various sources. On performing analysis, clash in schedules among stakeholders are identified and appropriate rescheduling is accomplished [4].

\section{B. Construction Scheduling}

As discussed, construction scheduling is a novel feature of BIM and is performed by analyzing live-data acquired through various sources. On performing analysis, clash in schedules among stakeholders are identified and appropriate rescheduling is accomplished [4].

\section{Cost Estimation}

Information on transaction, logistics and construction, onsite and off-site, transfers to the BIM platform and costs are monitored from data acquired based on management in realtime [5]. This can be integrated on various levels to monitor cash-flow.

\section{APPLICATIONS OF SENSOR TECHNOLOGIES IN DATA ACQUISITION}

Development in sensor technologies has been exponential over the past decade. However, its application in building construction sector has been relatively slow compared to other production industries. There are four types of on-site data acquisition requirements: (1) Positioning, (2) Tracking, (3) Progress Monitoring and (4) Quality Control. This section covers the potential sensor technologies with applications in building construction [6].

\section{A. Positioning}

Positioning is used in prefabrication as well as site assembly. Components are manufactured with great precision and demands an accuracy of up to a millimeter. The manufactured components are further assembled on site by referencing positions of the components with respect to the 3D BIM. Speed and accuracy are the important factors in positioning. Each building component would be tagged for a distinct location within the site and then the right position at the given location. Positioning is done using multiple coordinates and axial parameters for both position and orientation. The modern sensor technologies with an application in positioning include total stations (digital theodolites or tachometers), Global Navigation Satellite System (GNSS), laser planes, levels, and plummets. Laser and optical tachymeters are favored due to its accuracy. 


\section{1) Laser Tachymeters and Trackers}

Laser tachymeters are widely used in construction sites. It contains an electronic measuring device (EDM) combined with a theodolite which collectively functions as a total station. Tachymeters are capable of measuring two directional angles and distance to tagged objects and achieves positioning of tagged objects up to a millimeter accuracy.

Laser tachymeters use laser interferometer technology for positioning although the functioning is similar to conventional tachymeters. Trackers can be either manually operated or in more advanced systems tagged to a moving object. They are widely used in aerospace industry for positioning in large 3D spaces. Faro, Leica, and API are some of the trackers used in the industry.

\section{2) Optical Trackers}

6D positioning technology using cameras and optical trackers constitute this method of positioning. 6D positioning involves three parameters for positioning and three parameters for orientation. This optical technology helps in gaining accuracy of up to one micrometer up to a range of ten meters using cameras and optical triangulation method. NDI Optotrak is one such positioning device.

\section{3) Laser Indoor GPS}

Commonly used in a large-scale industrial space, this technology works very similar to GPS. It helps in positioning objects within a large area to an accuracy less than a millimetre. It uses rotating infrared lasers in the satellite to map the given site or space. This technology can be used to position other measuring devices within the site to map the overall data acquired. It is however more expensive than tachymeters or optical trackers. Nikon iSpace is one such product.

\section{4) Global Navigation Satellite System (GNSS); GPS}

GPS (Global Positioning System) technology is commonplace in everyday life and in construction. It is a type of GNSS that can pinpoint receivers anywhere on the globe. Although extensively used in tracking, its application in positioning will bring forth major breakthroughs in the construction industry.

\section{B. Tracking}

Tracking helps in better management. Applications of tracking includes (1) tracking of the materials and components from prefabrication environment to site, (2) tracking of machinery, assets and building components in site and (3) tracking of personnel on-site. Tracking is required only to know the relative positions and does not call for high speed or accuracy. Accuracy up to a meter is considered to be sufficient to satisfy its functions. The technologies adapted for tracking can grouped into three types: (1) radio frequencybased systems, (2) inertial based systems and (3) camerabased systems.

\section{1) RF-based Systems}

$\mathrm{RF}$ based systems in its most basic form may involve a WiFi or Bluetooth positioning system using Received Signal Strength Indication (RSSI) for tracking or in more complex situations may involve an RFID tag associated with each component, asset, or personnel. An RFID tag includes a transmitter which starts transmitting when the device is under an electromagnetic field, a storage that carries information of the component and a power source that converts the electromagnetic energy to power the device. Beacons or receivers are placed at distinct points in the site and the strength of the signal being transmitted as received at various beacons is used to calculate its position. Ubisense and nanoLoc offer sensor systems that calculate the location of an object using a radio propagation model.

\section{2) Inertial-based Systems}

Inertial based systems depend on accelerometers and gyrometers for tracking. Integration of signals in this case might cause drifting errors which result in reduction in accuracy. Development in MEMS (Micro Electro Mechanical System) sensor technology offers cheap and light weighted systems although the accuracy is compromised by default.

\section{3) Camera-based Systems}

Camera based systems use multiple CCD (Close-Circuit Device) cameras to observe selected features of an object such as edges, holes or tags. The processed images from these cameras are used to understand the image in 3D based on an algorithm. Since it is an optical system, proper lighting and calibration is required and setting up would consume time and effort. However, the system is more primitive and has been used for a long period.

\section{Progress Monitoring}

Progress monitoring conventionally deals with on-site measurements of built environment and its comparison with the drawings. Onset of BIM into the industry helped in creating a database for site updates and comparing it with the corresponding drawings or data. In an automated scenario, images are captured real-time at the site, and it's compared with virtual images created on BIM. It helps in automating the updating process and any discrepancies are distinguished by color coding. Implementing robotic technologies can reduce errors and structures can be built with 'zero-defect', which is a major sustainability principle. Construction practices could be continually reviewed, planned and monitored based on their capability to meet the projected state and implied requirement.

\section{1) RFID and CAD Integration}

In this monitoring system the components are provided with RFID tags. Data regarding the component is extracted from the BIM and compared with the updated RFID inspection data.

\section{2) Vision-based Monitoring}

Vision-based monitoring involves tracking down components, equipment and workers using real-time imagery. Cost estimation is extracted from the 3Dcoordinated data, using images from multiple camera sources and on further comparison with the construction schedule.

\section{3) Digital Photos}

Taking images and subjecting them to manual inspection is the conventional method. Advancements in technology enables automation of this process. A sequence of images is used can track progress in real-time. Images are further tagged to relevant CAD drawings using picture archives. 


\section{Quality Control}

Quality control is a continuous process to ensure adherence of components and finished products to the quality criteria and standards enforced by stakeholders. This section is concerned about collecting real-world data. It is important to integrate construction procedures with quality assurance processes.

\section{1) Imaging Systems, Shape Measurements and Scanners}

Laser-based imaging systems are used to gather real-world data in 3D. Imaging systems such as laser detection, laser radar and laser detection and ranging (LIDAR) systems are employed. Quality control gives sufficient weightage to speed and accuracy. The foresaid technologies are capable of producing high quality 3D images at high-speed although post processing phase, which involves comparison with the $3 \mathrm{D}$ model becomes time consuming, depending on the level of detail in the model.

Photogrammetric light measurement systems involve a light pattern projected on an object. The deviations in light patterns are recorded on camera and processed to prepare 3D images. Kinect sensor is a consumer-grade range camera with a high quality to price ratio. It works on triangulation principle and gives high quality images in depth and colour with a frame rate of $30 \mathrm{fps}$. It has an integrated light emitter which produces random light patterns.

\section{INFORMATION TECHNOLOGY (IT) AND SOFTWARE- BASED AUTOMATION}

The developments in sensor technology can be exploited in automation, only if the necessary software packages are designed and developed [7].

Software can be modified or developed to customize working in areas where the standard procedure is not followed. This opens possibility for human interventions to bring an element of creativity which might get lost in standardization.

Chen et al. [8] discusses an initiative termed as IRP (Incentive Reward Program). Under this initiative, workers causing least amount of wastage of construction material would be rewarded an incentive. This would be performed using bar-coding of materials and remotely tracking laborer's progress using pre-installed devices. An experiment was conducted to prove its relevance in a residential project in Hong Kong and was proved effective.

Halfway [9] proposes a software that could integrate information and knowledge to measure the decay in a building. He debates the relevance of having a building knowledge modelling (BKM) software as opposed to a building information modelling (BIM) software.

Project Performance Monitoring System (PPMS) proposed by Cheung et al. [10], provides a gateway for data acquired through project monitoring to be viewed and analyzed. The software is capable of automating monitoring of the construction project. Various parameters like people, cost, time, health and safety, quality, environment, client satisfaction and communication are laid out in the PPMS. Methods are devised for measuring performance based on each parameter and performance computing systems are established.
Irizarry et al. [11] proposed an IT-based solution for integrating the elements of construction supply chain management (CSCM). By integrating BIM with the Geographic Information System (GIS), the materials could be tracked, and timely on-site delivery can be ensured. It extends from the design phase to the construction phase and helps in making informed pre-design decisions based on suppliers' resource availability. It is capable of monitoring the available resources, controlling the material flow and visually representing the supply chain.

\section{FACTORS RESTRAINING AUTOMATION IN CONSTRUCTION INDUSTRY}

Introduction of robotic machinery into the construction site has raised safety concerns in the past. Non-adherence of the robotic machinery to the construction and building codes results in resistance from the building and safety authorities.

Lack of standardization and rise of innovative nonconventional design forms result in the industry's rejection of automation. With majority of the construction stakeholders being trained in a single trade, introduction of automation would require them to have retraining to run operations. This scenario raises concern among stakeholders. The following is the list of barriers that discourage contractors from implementing automation on construction site [12];

1. Lack of awareness about the necessity for automation;

2. High cost of acquisition, maintenance and updating;

3. Incompatibility with the conventional practices and construction operation;

4. Low technology literacy;

5. Lack of local availability and high cost of transportation;

6. Unacceptance from workers and management;

7. Variability in nature of construction processes.

Failure in attempts to commercialize automation can be explained by lack of customer interest, triggered by huge tolerance range, low levels of standardization and prefabrication, and medium level of industrialization [13].

\section{APPLICATIONS OF AUTOMATION IN BUILDING CONSTRUCTION}

The building construction processes can be classified into on-site and off-site works. While most construction processes in conventionally happens on site, the developments in fields of BIM-oriented design and IT have made it possible to shift work packages off-site. This progress led to the increased adoption of practices such as pre-fabrication, modularization, pre-assembly and off-site fabrication.

\section{A. Prefabrication}

Prefabrication includes the manufacturing and assembly of components in a factory or a manufacturing site and transporting complete assemblies or sub-assemblies to the construction site. In comparison to the conventional construction process where raw materials are procured and assembled on site, prefabrication process calls for industrialization of production. Increasing pre-work can help in product delivery with efficient control on cost and schedule, thus saving time and money in construction. Since the production takes place at industrial scales, wastage will 
be minimized. The Future Home project was an attempt at integrating construction automation in all stages of house building process.

\section{B. Civil Engineering}

In the context of construction, civil engineering refers to the ground processes of excavation, earth moving and piling for foundation that takes place before construction. Automation is achieved by creating a seamless interface between $\mathrm{CAD}$ and robotic operation. A robotic earthmoving equipment, designed by Carnegie Mellon University, automates the task of mass excavation. The software decides where to dig and where to dump in the most efficient way using laser rangefinders. Application of GPS enabled positioning and navigating excavators to targets with an accuracy of 25 millimeters.

\section{Foundation Construction}

Foundations are constructed by connecting prefabricated blocks in a straight line and corners using forklifts and backhoes. This has helped in speeding up the process by more than half and has reduced construction wastage since molds can be avoided.

\section{Frame Erection}

Conventional building frames are composed of reinforced concrete, steel frame or steel framed reinforced concrete. The automated systems pertaining to each of this classification is different and equally advanced. Reinforced concrete is the most popular due to the less expensive constituents.

Automated Building Construction System (ABCS) integrates the construction operation with factory automation. The material delivery system transports the building structural components from site delivery area to construction operation level with the help of a network of horizontal and vertical transportation system. The factory unit is vertically mobile and supported on alternate structural columns and supports the crane, material hoists and a structural steel roof for the finished structure. On completing every two floors the factory unit is automatically jacked up to the next level using a hydraulic connection mechanism. ABCS offers a super construction factory with warehouse facilities, automated conveyor systems and a centralized computer-controlled system.

Alternatively, the Big Canopy system by Obayashi works by assembling pre-cast members. The prefabricated units of beams, columns, slabs, and interior walls are sub-assembled and brought to site.

In brick assembly, EU based Rocco devised an automated robotic system with a wide range of 10 meters. The robot is equipped with a laser-tracked telemeter and follows the assembly sequence provided by a planning software. Except for an initiation process to know the brick palette position the process is completely automated.

\section{CONCLUSION}

While automation has established applications in distinct construction processes, there has been no recorded attempt at complete site automation. While robotics has picked up gradual pace by catering to specific processes, the sensor technology is getting explored in the construction industry for its specific applications in relevant areas. Various types of sensors which work in different conditions to cater to distinct situations were discussed. The software packages that facilitate construction and construction management are continuously evolving to achieve complete site automation. 4D BIM is a recent addition to the construction industry in a select few countries and 5D BIM is under development for commercial usage. Furthermore, it has been stated that a combination of the technologies discussed and illustrated through case examples brings efficiency to construction process by reducing time, cost and energy consumption. The significance of interoperability has been discussed and fast and timely data entry helps in improving this communication.

\section{REFERENCES}

[1] X. Wang, P. Ed Love, P. Davis. BIM+AR: a framework of bringing BIM to construction site. Constructopm Research Congress ASCE, 2012: 1175-1181

[2] T. Bock. The future of construction automation: Technological disruption and the upcoming ubiquity of robotics. Automation in Construction; 2015; 59.

[3] C. Z. Li. An Internet of Things-enabled BIM platform for on-site assembly services in prefabricated construction. Automation in Construction, 2018. 2018;89.

[4] H. Kim, K. Anderson. Generating construction schedules through automatic data extraction using open BIM technology. Automation in Construction. 2013; 35: 295-289.

[5] K. S. Lee, R. Kim, J. H. Yu. BIM and ontology-based approach for building cost estimation. Automation in Construction. 2014; 41: 96105.

[6] P. Vähä, T. Heikkilä, P. Kilpeläinen, M. Järviluoma, E. Gambao. Extending automation of building construction - Survey on potential. Automation in Construction. 2013; 36.

[7] R. Singh, A. Gehlot, V. P. Singh, V. Garg, S. Kumar, S. Choudhury, R. Pachauri. Role of automation in construction industries: a review. Journal of Engineering Technology, ASEE. 2017; Vol 6(2).

[8] Chen, Zhen, H. Li, C. T. Wong. An application of bar-code system for reducing construction wastes. Automation in Construction. 2002; Vol 11(5): 521-533.

[9] Halfawy, Mahmoud, T. Froese. Building Integrated Architecture/Engineering/Construction Systems Using Smart Objects. Journal of Computing in Civil Engineering. 2005; Vol 19(2): 172-181.

[10] Cheung, S. On, H. C. Suen, K. K. Cheung. PPMS: a web-based construction project performance monitoring system. Automation in Construction. 2004. Vol 13(3): 361-376.

[11] Irizarry, Javier, E. P. Karan, F. Jalaei. Integrating BIM and GIS to improve the visual monitoring of construction supply chain management. Automation in Construction, 2013; Vol 31: 241-254,

[12] Z. Strukova, M. Liska. Application of automation and robotics in construction work execution. Journal of Interdisiplinary Research, Technical University of Kosic. Faculty of Civil Engineering, Slovakia. 2014.

[13] V. Pentti, T. Heikkilä, P. Kilpeläinen, M. Järviluoma, R. Heikkilä. Survey on automation of the building construction and building products industry. VTT Technology. 2013.

[14] CII, Prefabrication, preassembly, modularisation and offsite fabrication in industrial construction: a framework for decision making. Construction Industry Instititute. 2002; 171-1. 